Palavras chave:

Dinâmica de carbono

Sucessão florestal

Mata Atlântica

Histórico:

Recebido 04/05/2012

Aceito $31 / 08 / 2014$

Keywords: Carbon dynamics Forest succession Atlantic Rainforest

Correspondência: liviatmfigueiredo@gmail.com
Lívia Thais Moreira de Figueiredo', Carlos Pedro Boechat Soares', Agostinho Lopes de Sousa', Hélio Garcia Leite', Gilson Fernandes da Silva²

\section{DINÂMICA DO ESTOQUE DE CARBONO EM FUSTE DE ÁRVORES DE UMA FLORESTA ESTACIONAL SEMIDECIDUAL}

RESUMO: Objetivou-se, neste trabalho, avaliar a dinâmica do estoque de carbono no fuste das árvores de uma Floresta Estacional Semidecidual, por meio do monitoramento de 10 parcelas permanentes instaladas na Mata da Silvicultura, Viçosa, Minas Gerais. Foram identificados e medidos todos os indivíduos com DAP $\geq 5 \mathrm{~cm}$, nos anos de 1994, 1997, 2000, 2004 e 2008. O estoque médio de carbono no fuste das árvores aumentou de 47,9 ton $\cdot \mathrm{ha}^{-1}$, no ano de 1994, para 6I,8I ton $\cdot \mathrm{ha}^{-1}$, no ano de 2008, representando, para os 14 anos de monitoramento, um incremento periódico de 0,994 ton $\cdot \mathrm{ha}^{-1} \cdot \mathrm{ano}^{-1}$.

\section{CARBON STOCK DYNAMICS OF TREE STEMS IN A TROPICAL SEMIDECIDUOUS FOREST}

ABSTRACT: The objective of this study was to evaluate the dynamics of stem carbon storage in a Semideciduous Forest, through the monitoring of 10 permanent plots established in the Mata da Silvicultura, Viçosa, Minas Gerais. All trees with DBH $\geq 5 \mathrm{~cm}$ were identified and measured in the years 1994, 1997, 2000, 2004 and 2008. The stems average carbon storage increased 47.9 ton $\cdot \mathrm{ha}^{-1}$ from the year of 1994 to $6 \mathrm{I} .8 \mathrm{I} \mathrm{ton} \cdot \mathrm{ha}^{-1}$ in the year of 2008, representing, for the monitoring of I 4 years, a regular increase of 0.994 ton $\cdot$ ha $^{-1} \cdot$ year'. 


\section{INTRODUÇÃO}

A Floresta Atlântica, um dos mais importantes ecossistemas brasileiros, é, atualmente, representada por fragmentos de formações florestais em estágio secundário de sucessão (CONSERVATION INTERNATIONAL DO BRASIL, 2000). Essas formações florestais, apesar de fragmentadas, ainda abrigam grande riqueza de fauna, flora e desempenham papel fundamental no clima e no ciclo do carbono (VIEIRA et al., 2005). As florestas capturam dióxido de carbono $\left(\mathrm{CO}_{2}\right)$ da atmosfera, por meio da fotossíntese e armazenam o carbono em sua biomassa, principalmente na madeira. Segundo Fonseca et al. (20I I), as florestas também perdem carbono para a atmosfera através da respiração das plantas e da decomposição de material orgânico, no entanto, essas perdas, geralmente, são menores do que os ganhos em carbono.

Segundo Hoover et al. (20I2), embora o sequestro de carbono tenha se tornado um objetivo de gestão da floresta, o potencial de armazenamento de carbono dessas florestas ainda não é bem compreendido. Diversos estudos foram realizados com o objetivo de quantificar o estoque de carbono em florestas naturais (AMARO, 20I0; BRITEZ et al., 2006; WATZLAWICK et al., 2002), no entanto, estes estudos se referem ao carbono estocado em períodos específicos, e não consideram o estoque ao longo dos processos sucessionais.

Assim, o que se observa é que, pela necessidade de se controlar o aumento nos níveis de $\mathrm{CO}_{2}$ atmosférico, aliado à característica das espécies em acumular carbono em seus tecidos, aumentou a demanda por estudos sobre a dinâmica de florestas naturais, com o objetivo de quantificar o carbono estocado pelas espécies ao longo dos processos de sucessão, a fim de que estes estudos sirvam de base para $\circ$ manejo e conservação desses remanescentes florestais nativos.

Brun (2007) ressalta que, em estudos dessa natureza, tão importante quanto avaliar o acúmulo de biomassa ou carbono na floresta natural como um todo, é avaliar também o potencial em sequestrar e acumular carbono de cada espécie individualmente. Nesse sentido, objetivou-se, neste trabalho, avaliar a dinâmica do carbono estocado no fuste das árvores de uma Floresta Estacional Semidecidual no município de Viçosa, Minas Gerais.

\section{MATERIAL E MÉTODOS}

\section{Caracterização da área de estudo}

O presente estudo foi conduzido em um fragmento de 17 ha, de Floresta Estacional Semidecidual
Montana (VELOSO et al., 199I), conhecido como Mata da Silvicultura, localizado no campus da Universidade Federal de Viçosa, município de Viçosa, Minas Gerais

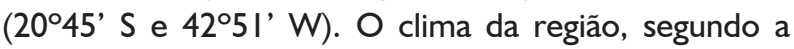
classificação de Köppen, é do tipo Cwa, mesotérmico úmido com verões chuvosos e invernos secos (VIANELLO; ALVES, 1991).

A topografia local é acidentada, com vales estreitos e úmidos, com a altitude variando aproximadamente, de 600 a 970 metros, estando o município situado 649 metros de altitude. Os solos são predominantemente o Latossolo Álico, nos morros e encostas e o Vermelho-Amarelo Câmbico nos terraços (CORREA, 1984).

\section{Coleta e análise dos dados}

Os dados utilizados neste trabalho foram coletados em 10 parcelas retangulares de área fixa de $20 \mathrm{~m} \times 50 \mathrm{~m}$ (0,I ha). Em cada parcela, todos os indivíduos arbóreos com DAP $\geq 5 \mathrm{~cm}$, foram medidos e identificados.

A identificação do material botânico coletado foi feita por meio de comparações com o material do Herbário do Departamento de Biologia Vegetal da UFV e de consultas a especialistas e literatura especializada. Para a confirmação dos nomes das espécies e de seus autores foram feitas consultas ao site do Missouri Botanical Garden (20II).

No estudo da composição florística, os indivíduos amostrados foram classificados em categorias sucessionais de acordo com a divisão proposta por Gandolfi et al. (1995), segundo a qual, as espécies são classificadas em: pioneiras $(\mathrm{PI})$, secundárias iniciais $(\mathrm{SI})$, secundárias tardias (ST) e espécies sem classificação (SC).

Após o primeiro levantamento realizado em 1994, todas as parcelas foram remedidas nos anos de 1997, 2000, 2004 e 2008. Em cada remedição, todos os indivíduos que tivessem DAP maior ou igual a $5 \mathrm{~cm}$ foram medidos, identificados e contabilizados como ingresso.

O carbono estocado no fuste de cada indivíduo amostrado nos levantamentos ( 1994 - 2008) foi estimado por meio da equação 1 , desenvolvida por Amaro (2010) em um estudo realizado na mesma área, sendo $C F c c=$ carbono no fuste com casca, em $\mathrm{kg}$; DAP = diâmetro medido a $1,30 \mathrm{~m}$ de altura, em $\mathrm{cm}$; e $\mathrm{Hf}=$ altura do fuste, em $\mathrm{m}$.

CFCC $=0,013840 \cdot D A P^{2,437632} \cdot H f^{0,428609}$

$\overline{\mathrm{R}}^{2}=95,34 \% \quad S_{y, x}= \pm 23,6 \%$ 
Para verificar diferenças significativas entre os estoques de carbono nos inventários sucessivos (19942008), ajustou-se uma equação linear simples (equação 2), sendo $Y_{i}=$ estoque de carbono no levantamento $i$; $Y_{j}=$ estoque de carbono no levantamento $j ; \beta_{0}$ e $\beta_{1}=$ parâmetros do modelo; $\varepsilon=$ erro aleatório.

$Y_{i}=\beta_{0}+\beta_{1} \cdot Y_{j}+\varepsilon$

A hipótese de igualdade entre as estimativas foi verificada considerando $\beta=\left[\begin{array}{l}0 \\ 1\end{array}\right]$ empregando-se o teste $F$ de Graybill (1976) dada pela equação 3, com o valor tabelado de $\mathrm{F}_{5 \%}(2, \mathrm{n}-2)$, sendo $Y_{1}=$ vetor do estoque de carbono nos levantamentos realizados (comparados 2 a 2); QMRes = quadrado médio do resíduo da regressão.

$F\left(H_{0}\right)=\frac{(\beta-\theta)^{\prime} \cdot\left(Y_{1}^{\prime} \cdot Y_{1}\right) \cdot(\beta-\theta)}{2 \cdot \text { QMRes }}$

Para o cálculo do ingresso em número de fustes e em carbono estocado por hectare, por período de monitoramento, foram consideradas as árvores vivas que, em uma ocasião de monitoramento, apresentavam DAP inferior a $5 \mathrm{~cm}$ e que na ocasião seguinte apresentavam DAP igual ou superior a $5 \mathrm{~cm}$. Para os quatorze anos de monitoramento (1994 2008), considerou-se como ingresso, as árvores que foram classificadas como ingresso em alguma época do monitoramento e que, no inventário realizado em 2008, foram classificadas como sobreviventes. No cálculo da mortalidade em número de fustes e em carbono estocado por hectare, por período de monitoramento, foram considerados como mortos os fustes medidos em uma ocasião e que se encontravam mortos na ocasião seguinte.

As estimativas de incremento bruto e líquido em carbono estocado no fuste por hectare para cada uma das ocasiões de monitoramento foram obtidas, utilizando-se as equações 4, 5, 6 e 7 (DAVIS; JOHNSON, 1987), sendo $\mathrm{lb}=$ incremento bruto, excluindo o ingresso; $l b i=$ incremento bruto, incluindo o ingresso; $I L=$ incremento líquido, excluindo $\circ$ ingresso; $I L i=$ incremento líquido, incluindo o ingresso; $C_{f}=$ carbono (ton $\cdot \mathrm{ha}^{-1}$ ) das árvores remanescentes (árvores vivas nos dois inventários) no final do período; $C_{i}=$ carbono (ton. $\mathrm{ha}^{-1}$ ) das árvores remanescentes (árvores vivas nos dois inventários), no início do período; $M=$ mortalidade $\left(\right.$ ton $\cdot$ ha $^{-1}$ ) no período; $I$ = ingresso em carbono $\left(\right.$ ton $\cdot h a^{-1}$ ) no período.

$I b=\left(C_{f}-I\right)-\left(C_{i}-M\right)$

$$
\begin{aligned}
& I b i=C_{f}-\left(C_{i}-M\right) \\
& I L=\left(C_{f}-I\right)-C_{i} \\
& I L i=C_{f}-C_{i}
\end{aligned}
$$

As estimativas de incremento em carbono estocado no fuste (ton $h^{-1}{ }^{-1}$ ) para o período total de monitoramento (1994-2008) foram feitas com base nas mesmas expressões, porém considerou-se a mortalidade acumulada nos 14 anos de monitoramento e o ingresso apenas das árvores remanescentes no final do período, conforme adotado por Ferreira et al. (1998) em sua pesquisa.

As estimativas de incrementos periódicos líquidos anuais, por espécie, e por ocasião de monitoramento, foram obtidas pela equação 8 , sendo $I P A_{l i}=$ incremento periódico líquido anual; $I L i=$ incremento líquido, incluindo o ingresso; $t=$ intervalo de tempo, em anos.

$I P A_{l i}=\frac{I L i}{t}$

\section{RESULTADOS}

\section{Caracterização da área de estudo}

A área de estudo, pertencente a um fragmento de Floresta Estacional Semidecidual Montana (VELOSO et al., 1991), encontra-se, com base nos valores de diâmetro médio (q) e altura total média observados (Tabela 1), em sucessão secundária de estágio médio (BRASIL, 2007), sendo que, apenas uma parcela (7) atingiu o estágio avançado a partir do terceiro ano de monitoramento.

TABELA 1 Caracterização dendrométrica, número de indivíduos e de fustes na Mata da Silvicultura, Viçosa, Minas Gerais, no período de 1994 a 2008.

TABLE 1 Dendrometric characterization, number of individuals and stems in the "Mata da Silvicultura", Viçosa, Minas Gerais, in the period 1994 to 2008

\begin{tabular}{lccccc}
\hline \multirow{2}{*}{ Parâmetro } & \multicolumn{5}{c}{ Ano de monitoramento } \\
\cline { 2 - 6 } & 1994 & 1997 & 2000 & 2004 & 2008 \\
\hline Indivíduo (por hectare) & 1379 & 1383 & 1331 & 1307 & 1326 \\
Fuste (por hectare) & 1521 & 1540 & 1497 & 1473 & 1492 \\
DAP médio q (cm) & 14,26 & 14,69 & 14,99 & 15,09 & 15,37 \\
Altura total média (m) & 10,45 & 11,11 & 11,44 & 12,49 & 12,49 \\
Altura do fuste média $(\mathrm{m})$ & 6,98 & 7,31 & 7,50 & 7,46 & 7,95 \\
\hline
\end{tabular}


O número de famílias e de gêneros permaneceu praticamente constante de 1994 a 2008 (Tabela 2). No último período de medição, houve o maior aumento no número de espécies na área, que passou de um total de 138 espécies no ano de 2004, para 153 espécies em 2008. Esse aumento se deve, principalmente, ao ingresso de novas espécies na área, em função do avanço da sucessão.

TABELA 2 Número de famílias, gêneros e espécies encontradas na Mata da Silvicultura no período de 1994 a 2008.

TABLE 2 Number of families, genera and species found in the "Mata da Silvicultura" in the period 1994 to 2008

\begin{tabular}{lccccc}
\hline \multirow{2}{*}{ Parâmetro } & \multicolumn{5}{c}{ Ano de monitoramento } \\
\cline { 2 - 6 } & 1994 & 1997 & 2000 & 2004 & 2008 \\
\hline Famílias & 43 & 43 & 43 & 42 & 42 \\
Gêneros & 100 & 98 & 99 & 99 & 103 \\
Espécies identificadas & 136 & 135 & 137 & 135 & 148 \\
Espécies não identificadas & 4 & 3 & 4 & 3 & 5
\end{tabular}

As famílias mais representativas em número de espécies encontradas no fragmento foram: Leguminoseae, Euphorbiaceae, Myrtaceae, Lauraceae, Rubiaceae e Flacourtiaceae. Os gêneros com o maior número de espécies encontrados na área foram: Ocotea, Casearia, Inga, Machaerium, Miconia, Myrcia, Psychotria.

A área apresenta dominância de espécies secundárias iniciais e tardias. Ao longo dos levantamentos realizados, foi possível observar que houve uma redução no número de espécies pioneiras e um aumento no número de espécies secundárias iniciais, secundárias tardias e sem classificação na área de estudo.

\section{Estoque de carbono no fuste das árvores}

De acordo com a Tabela 3, houve aumento no estoque de carbono no fuste das árvores de 1994 a 2008, de 47,90 ton $\cdot h^{-1}$ para 61,81 ton $\cdot h a^{-1}$. Com base no teste $F$ modificado de Graybill, verificou-se que, apenas no período de 2000 a 2004, não houve diferença estatística entre os estoques de carbono no fuste das árvores.

O maior estoque de carbono na parcela 7 pode ser explicado pelo fato dessa parcela se encontrar em estágio mais avançado de sucessão. Segundo Watzlawick et al. (2002), o maior estoque de carbono em estágios mais avançados de sucessão ocorre em função de, neste estágio, ocorrerem indivíduos de maior porte, além de uma composição florística mais rica em espécies, com madeiras de maior densidade.
TABELA 3 Estoque de carbono no fuste das árvores (ton $\cdot$ ha $^{-1}$ ) por parcela e por ocasião de monitoramento na Mata da Silvicultura, Viçosa, Minas Gerais e teste F modificado de Graybill.

TABLE 3 Carbon storage in the stems of trees (ton ha $^{-1}$ ) per plot and during monitoring in the "Mata da Silvicultura", Viçosa, Minas Gerais and Graybill's modified $\mathrm{F}$ test.

\begin{tabular}{|c|c|c|c|c|c|}
\hline \multirow{2}{*}{ Parcela } & \multicolumn{5}{|c|}{ Ano de monitoramento } \\
\hline & 1994 & 1997 & 2000 & 2004 & 2008 \\
\hline 1 & 70,90 & 75,93 & 80,62 & 79,84 & 75,22 \\
\hline 2 & 31,09 & 35,62 & 38,19 & 39,01 & 44,42 \\
\hline 3 & 44,42 & 48,81 & 50,85 & 38,06 & 41,93 \\
\hline 4 & 29,98 & 34,11 & 29,98 & 28,58 & 32,20 \\
\hline 5 & 36,38 & 41,82 & 42,77 & 44,61 & 47,03 \\
\hline 6 & 49,60 & 54,21 & 56,28 & 59,29 & 65,81 \\
\hline 7 & 89,95 & 106,72 & 110,65 & 113,71 & 132,07 \\
\hline 8 & 42,82 & 48,02 & 49,43 & 51,58 & 57,89 \\
\hline 9 & 35,39 & 37,39 & 41,33 & 44,07 & 50,25 \\
\hline 10 & 48,50 & 59,76 & 61,71 & 66,16 & 71,29 \\
\hline Média & 47,90 & 54,24 & 56,18 & 56,49 & 61,81 \\
\hline \multirow[t]{2}{*}{ Desvio-padrão } & $\pm 1,90$ & $\pm 2,24$ & $\pm 2,38$ & $\pm 2,51$ & $\pm 2,83$ \\
\hline & \multicolumn{5}{|c|}{ Teste F de Graybill } \\
\hline Período & $\begin{array}{c}1994 \\
- \\
1997 \\
\end{array}$ & $\begin{array}{c}1997 \\
- \\
2000 \\
\end{array}$ & $\begin{array}{c}2000 \\
- \\
2004 \\
\end{array}$ & $\begin{array}{c}2004 \\
- \\
2008 \\
\end{array}$ & $\begin{array}{c}1994 \\
- \\
2008 \\
\end{array}$ \\
\hline $\mathrm{F}\left(\mathrm{H}_{\mathrm{o}}\right)$ & $26,49^{*}$ & $5,29^{*}$ & $0,14^{\mathrm{ns}}$ & $6,31^{*}$ & $10,93^{*}$ \\
\hline
\end{tabular}

$\mathrm{F}_{\text {Tabelado }}=4,45 ;$ ns $=$ não significativo a $5 \%$ de significância; * $=$ significativo a $5 \%$ de significância.

O estoque de carbono encontrado neste trabalho, no último ano de medição, foi próximo ao encontrado por Amaro (2010), em um trabalho realizado na Mata da Silvicultura, que foi de, em média, 58,2 toneladas de carbono por hectare estocado no fuste com casca dos indivíduos arbóreos vivos.

Em um estudo realizado em uma floresta madura no município de Viçosa, Minas Gerais, Ribeiro et al. (2009) encontrou estimativas de carbono estocado no fuste sem casca das árvores iguais a 83,34 ton $\cdot \mathrm{ha}^{-1}$, valor superior aos encontrados neste trabalho.

Em relação a outros ecossistemas pertencentes ao domínio da Mata Atlântica, os valores encontrados neste trabalho foram inferiores ao encontrado por Brun (2004) que encontrou 65,4 toneladas de carbono por hectare na biomassa acima do solo em uma Floresta Estacional Decidual na região de Santa Tereza (RS).

Watzlawick et al. (2002), estudando a fixação de carbono em floresta Ombrófila Mista, em diferentes 
estágios de regeneração, encontraram estoque de carbono de 28,84 ton $\cdot$ ha $^{-1}$ na biomassa acima do solo em floresta em estágio inicial de regeneração; 70,23 ton $\cdot \mathrm{ha}^{-1}$ em estágio médio; e 165,02 ton·ha-1 para a floresta em estágio avançado de regeneração.

A família Leguminoseae estocou, em média, 31,30 ton $\cdot$ ha $^{-1}$ de carbono no fuste das árvores, durante todo o período de monitoramento, valor superior a $50 \%$ do total de carbono estocado na área. Além da família Leguminoseae, as famílias Flacourtiaceae, Lauraceae, Euphorbiaceae, Cecropiaceae e Rubiaceae, também se destacaram em termos de estoque de carbono no fuste das árvores.

grupo das espécies secundárias iniciais foi o que apresentou maior estoque de carbono no fuste das árvores (Tabela 4). Em relação ao estoque absoluto, todas as categorias sucessionais apresentaram tendência a aumento na quantidade de carbono estocado no fuste das árvores durante o período de monitoramento. Em termos percentuais, apenas as espécies secundárias tardias apresentaram aumento na quantidade de carbono estocado no fuste das árvores.

Em um estudo visando a avaliar a dinâmica do carbono orgânico em espécies arbóreas em uma Floresta Estacional Decidual em Santa Tereza, RS, Brun (2007) constatou-se que, dentre os grupos sucessionais, o maior potencial de acúmulo para os galhos (vivos e mortos) e para a madeira, também foi apresentado pelas espécies secundárias iniciais.

Em relação ao ingresso e a mortalidade, observase que, o ingresso acumulado (1994 - 2008) foi de, em média, 328 fustes.ha-1 ( $1,57 \%$ ao ano) o que representou um ingresso em carbono igual a 0,90 ton $\cdot \mathrm{ha}^{-1}(0,10 \%$ ao ano). Já, a mortalidade acumulada nos quatorze anos de monitoramento foi de 4 I 3 fustes $h^{-1}$ ( I,87\% ao ano), ou seja, uma mortalidade igual a 10,17 ton $\cdot \mathrm{ha}^{-1}$ em carbono ( I,03\% ao ano).

\section{Incremento do estoque em carbono}

O primeiro (1994 a 1997) e o último (2004 a 2008) períodos de monitoramento, foram os que apresentaram maior incremento líquido incluindo o ingresso em carbono estocado no fuste das árvores, com 6,34 ton $\cdot \mathrm{ha}^{-1}$ e 5,32 ton $\cdot \mathrm{ha}^{-1}$, respectivamente (Tabela 5). $\mathrm{O}$ incremento líquido em carbono incluindo o ingresso para o período total de monitoramento foi de I3,9 I ton. $\mathrm{ha}^{-1}$, o que resultou em um incremento periódico anual em carbono de, em média, 0,99 ton $\cdot \mathrm{ha}^{-1} \cdot \mathrm{ano}^{-1}$.

Souza et al. (20l I), em estudo realizado em dois fragmentos de Floresta Estacional Semidecidual no Vale do Rio Doce, em Minas Gerais, no período de 2002 a 2007, encontrou no fragmento em estágio médio de regeneração crescimento periódico médio igual a 0,14 toneladas por hectare por ano de carbono no fuste das árvores, valor bem inferior ao encontrado neste trabalho. Para o fragmento em estágio avançado, o crescimento periódico médio foi igual a 0,92 toneladas por hectare por ano de carbono no fuste das árvores.

Para o período total de monitoramento, o grupo das espécies secundárias iniciais foi o que apresentou maior incremento periódico anual em carbono, incluindo o ingresso, 0,564 ton $\cdot \mathrm{ha}^{-1}$ (Tabela 5). O menor incremento periódico anual em carbono foi das espécies sem classificação, com 0,009 ton $\cdot$ ha $^{-1}$.

As espécies de maior incremento periódico líquido anual em carbono no fuste das árvores, incluindo o ingresso (IPAli) durante o período total de monitoramento (I994 a 2008) foram: Anadenanthera peregrina (L.) Speg. (SI); Newtonia contorta (DC.) Burkart. (ST); Apuleia leiocarpa (Vogel) J. F. Macbr. (SI); Casearia ulmifolia Vahl ex Vent. (SI); Bathysa nicholsonii K. Schum. (SI); Ocotea odorifera Rohwer. (ST); Cecropia hololeuca Miq. (PI); Casearia arborea (Rich.) Urb. (SI); Cariniana estrellensis (Raddi) Kuntze. (ST); e Platypodium elegans Vogel. (ST) (Tabela 6).

TABELA 4 Estoque de carbono no fuste das árvores, em ton ha-1 $\mathrm{e} \mathrm{em} \mathrm{\% ,} \mathrm{por} \mathrm{categoria} \mathrm{sucessional} \mathrm{(CS)} \mathrm{e} \mathrm{por} \mathrm{ano} \mathrm{de} \mathrm{monitoramento}$ na Mata da Silvicultura, Viçosa, Minas Gerais, no período de 1994 a 2008, em que: $\mathrm{PI}$ = pioneira; SI = secundária inicial; ST = secundária tardia; SC = sem classificação.

TABLE 4 Carbon Stock in the tree stems, in ton ha"-1 and \%, by successional category (CS) and per monitoring year in the "Mata da Silvicultura", Viçosa, Minas Gerais, in the period from 1994 to 2008, where: $\mathrm{PI}=$ pioneer; SI = early secondary; ST = late secondary; SC = unrated.

\begin{tabular}{|c|c|c|c|c|c|c|c|c|c|c|}
\hline \multirow{3}{*}{ CS } & \multicolumn{10}{|c|}{ Ano de monitoramento } \\
\hline & \multicolumn{2}{|c|}{1994} & \multicolumn{2}{|c|}{1997} & \multicolumn{2}{|c|}{2000} & \multicolumn{2}{|c|}{2004} & \multicolumn{2}{|c|}{2008} \\
\hline & ton ha-1 & $\%$ & ton ha-1 & $\%$ & ton ha-1 & $\%$ & ton $\mathrm{ha}^{-1}$ & $\%$ & ton $\mathrm{ha}^{-1}$ & $\%$ \\
\hline $\mathrm{PI}$ & 3,60 & 7,5 & 3,81 & 7,1 & 3,49 & 6,2 & 3,46 & 6,1 & 3,80 & 6,1 \\
\hline SI & 33,26 & 69,4 & 37,15 & 68,4 & 38,25 & 68,1 & 38,19 & 67,6 & 41,15 & 66,6 \\
\hline ST & 10,45 & 21,9 & 12,70 & 23,4 & 13,83 & 24,6 & 14,24 & 25,2 & 16,15 & 26,2 \\
\hline SC & 0,59 & 1,2 & 0,58 & 1,1 & 0,61 & 1,1 & 0,60 & 1,1 & 0,71 & 1,1 \\
\hline Total & 47,90 & 100 & 54,24 & 100 & 56,18 & 100 & 56,49 & 100 & 61,81 & 100 \\
\hline
\end{tabular}


TABELA 5 Incremento total bruto e líquido em carbono para cada período de monitoramento e por categoria sucessional (CS), em que: I - ingresso; $M$ - mortalidade; $I b i$ - incremento bruto, incluindo o ingresso; $I b$ - incremento bruto, excluindo o ingresso; $I L i$ - incremento líquido, incluindo o ingresso; IL - incremento líquido, excluindo o ingresso; IPAi - incremento periódico líquido; $\mathrm{PI}$ = pioneira; $\mathrm{SI}$ = secundária inicial; $\mathrm{ST}$ = secundária tardia; SC = sem classificação.

TABLE 5 Gross and net carbon increase for each monitoring period and category of succession (CS), where: $I$ - ingrowth; $M$ - mortality; Ibi - gross increase, including the ingrowth; Ib - gross increase, excluding the ingrowth; ILi - net increase, including the ingrowth; $I L$ - net increase, excluding the ingrowth; IPA $i$ - regular increase; PI = pioneer; SI = early secondary; $\mathrm{ST}=$ late secondary; $\mathrm{SC}=$ unrated.

\begin{tabular}{|c|c|c|c|c|c|c|c|c|c|}
\hline Período & Carbono final & I & Carbono inicial & $M$ & Ibi & Ib & ILi & IL & \multirow{2}{*}{$\begin{array}{c}\text { IPAi } \\
\left(\text { ton } \cdot \mathrm{ha}^{-1} \cdot \mathrm{ano}^{-1}\right)\end{array}$} \\
\hline & & & & & & & & & \\
\hline $94-97$ & 54,239 & 0,195 & 47,901 & 1,828 & 8,166 & 7,971 & 6,338 & 6,143 & 2,113 \\
\hline $97-00$ & 56,182 & 0,118 & 54,239 & 2,615 & 4,558 & 4,440 & 1,943 & 1,825 & 0,648 \\
\hline $00-04$ & 56,492 & 0,099 & 56,182 & 2,748 & 3,058 & 2,959 & 0,310 & 0,211 & 0,077 \\
\hline $04-08$ & 61,811 & 0,594 & 56,492 & 2,980 & 8,299 & 7,706 & 5,319 & 4,726 & 1,330 \\
\hline $94-08$ & 61,811 & 1,006 & 47,901 & 10,171 & 24,081 & 23,075 & 13,910 & 12,904 & 0,994 \\
\hline \multirow{2}{*}{$\begin{array}{c}\text { Categoria } \\
\text { sucessional } \\
(1994 \text { a 2008) } \\
\end{array}$} & Carbono final & I & Carbono inicial & $M$ & $I b i$ & $I b$ & ILi & IL & \multirow{2}{*}{$\begin{array}{c}\text { IPAi } \\
\left(\text { ton } \cdot \mathrm{ha}^{-1} \cdot \text { ano }^{-1}\right)\end{array}$} \\
\hline & & & & & & & & & \\
\hline $\mathrm{PI}$ & 3,798 & 0,125 & 3,600 & 1,033 & 1,232 & 1,107 & 0,199 & 0,073 & 0,014 \\
\hline SI & 41,155 & 0,660 & 33,261 & 7,220 & 15,113 & 14,453 & 7,893 & 7,234 & 0,564 \\
\hline ST & 16,149 & 0,141 & 10,453 & 1,761 & 7,457 & 7,316 & 5,697 & 5,555 & 0,407 \\
\hline SC & 0,708 & 0,079 & 0,587 & 0,157 & 0,279 & 0,199 & 0,121 & 0,042 & 0,009 \\
\hline Total & 61,811 & 1,006 & 47,901 & 10,171 & 24,081 & 23,075 & 13,910 & 12,904 & 0,994 \\
\hline
\end{tabular}

TABELA 6 Incremento em carbono no fuste das árvores (ton $\cdot$ ha-1 ${ }^{-1}$ por espécie em ordem decrescente de IPAli para o período total de monitoramento $(1994$ - 2008) na Mata da Silvicultura, Viçosa, Minas Gerais, em que: I - ingresso; $M$ - mortalidade; Ibi - incremento bruto, incluindo o ingresso; lb - incremento bruto, excluindo o ingresso; ILi - incremento líquido, incluindo o ingresso; IL - incremento líquido, excluindo o ingresso; IPAi - incremento periódico líquido

TABLE 6 Carbon increase in the tree stems (ton $\cdot$ ha $^{-1}$ ) by species in decreasing order of IPAli for the total monitoring period in the "Mata da Silvicultura", Viçosa, Minas Gerais, where: I - ingrowth; $M$ - mortality; Ibi - gross increase, including the ingrowth; $I b$ - gross increase, excluding the ingrowth; ILi - net increase, including the ingrowth; IL - net increase, excluding the ingrowth; IPAi - regular increase

\begin{tabular}{|c|c|c|c|c|c|c|c|c|c|}
\hline Espécie & $\begin{array}{l}\text { Carbono } \\
\text { final }\end{array}$ & I & $\begin{array}{c}\text { Carbono } \\
\text { inicial }\end{array}$ & $\begin{array}{l}M \\
\text { (ton } \mathrm{h}\end{array}$ & Ibi & $I b$ & ILi & IL & $\begin{array}{c}I P A i \\
\left(\text { ton } \cdot \text { ha }^{-1} \cdot \text { ano }^{-1}\right)\end{array}$ \\
\hline Anadenanthera peregrina (L.) Speg. & 16,639 & 0,144 & 12,818 & 1,671 & 5,492 & 5,348 & 3,821 & 3,677 & 0,273 \\
\hline Newtonia contorta (DC.) Burkart. & 7,425 & 0,007 & 4,035 & 0,185 & 3,576 & 3,569 & 3,391 & 3,384 & 0,242 \\
\hline Apuleia leiocarpa (Vogel) J. F. Macbr & 3,165 & 0,049 & 2,269 & 0,078 & 0,974 & 0,925 & 0,896 & 0,847 & 0,064 \\
\hline Casearia ulmifolia Vahl ex Vent. & 2,947 & 0,004 & 2,154 & 0,209 & 1,003 & 0,998 & 0,793 & 0,789 & 0,057 \\
\hline Bathysa nicholsonii K. Schum. & 1,565 & 0,071 & 0,854 & 0,063 & 0,775 & 0,704 & 0,712 & 0,641 & 0,051 \\
\hline Ocotea odorifera Rohwer. & 1,175 & 0,019 & 0,586 & 0,006 & 0,595 & 0,576 & 0,589 & 0,570 & 0,042 \\
\hline Cecropia hololeuca Miq. & 2,242 & - & 1,713 & - & 0,529 & 0,529 & 0,529 & 0,529 & 0,038 \\
\hline Casearia arborea (Rich.) Urb. & 1,406 & - & 0,986 & 0,131 & 0,550 & 0,550 & 0,420 & 0,420 & 0,030 \\
\hline Cariniana estrellensis (Raddi) Kuntze. & 0,714 & - & 0,331 & 0,011 & 0,394 & 0,394 & 0,383 & 0,383 & 0,027 \\
\hline Platypodium elegans Vogel. & 1,755 & 0,001 & 1,456 & 0,171 & 0,470 & 0,469 & 0,299 & 0,297 & 0,021 \\
\hline Nectandra lanceolata Nees. & 0,649 & 0,002 & 0,404 & 0,117 & 0,362 & 0,360 & 0,245 & 0,244 & 0,018 \\
\hline Sorocea bonplandii (Baill.) W. C. Burger & 0,424 & 0,008 & 0,202 & - & 0,222 & 0,214 & 0,222 & 0,214 & 0,016 \\
\hline Maprounea guianensis Aubl. & 0,487 & - & 0,273 & 0,004 & 0,217 & 0,217 & 0,213 & 0,213 & 0,015 \\
\hline Virola sebifera Aubl. & 0,321 & - & 0,115 & - & 0,206 & 0,206 & 0,206 & 0,206 & 0,015 \\
\hline Siparuna arianeae V. Pereira & 0,519 & 0,140 & 0,316 & 0,109 & 0,312 & 0,172 & 0,203 & 0,063 & 0,014 \\
\hline Sub-total & 41,432 & 0,445 & 28,510 & 2,755 & 15,677 & 15,232 & 12,922 & 12,477 & 0,923 \\
\hline Demais espécies & 20,379 & 0,561 & 19,391 & 7,416 & 8,404 & 7,843 & 0,988 & 0,427 & 0,071 \\
\hline Total & 61,811 & 1,006 & 47,901 & 10,171 & 24,081 & 23,075 & 13,910 & 12,904 & 0,994 \\
\hline
\end{tabular}


Ao todo, 32 espécies apresentaram incremento periódico líquido anual negativo em carbono no fuste, incluindo $\circ$ ingresso, sendo que destas, 7 (21,87\%) foram classificadas como pioneiras; 15 (46,88\%) como secundárias iniciais; 7 (21,87\%) como secundárias tardias e $3(9,38 \%)$ como espécies sem classificação.

\section{CONCLUSÕES}

Após as análises, pode-se concluir que: o estoque de carbono no fuste das árvores aumentou de 47,9 ton ha $^{-1}$, no ano de 1994, para 6I,8I ton ha ${ }^{-1}$, no ano de 2008, o que representou, em média, um incremento em carbono de 0,994 ton $\cdot$ ha $^{-1} \cdot$ ano $^{-1}$; a família Leguminoseae e as espécies secundárias inicias foram as mais representativas na área em termos de carbono estocado no fuste das árvores.

\section{REFERÊNCIAS}

AMARO, M. A. Quantificação do estoque volumétrico, de biomassa e de carbono em uma Floresta Estacional Semidecidual no município de Viçosa, MG. 2010. 168 f. Tese (Doutorado em Ciências Florestais) - Universidade Federal de Viçosa, Viçosa, 2010.

BRASIL. Resolução/CONAMA n 392, de 25 de junho de 2007. Definição de vegetação primária e secundária de regeneração de Mata Atlântica no estado de Minas Gerais. Diário Oficial [da] República Federativa do Brasil, Brasília, 26 jun. 2007.

BRITEZ, R. M.; BORGO, M.; TIEPOLO, G.; FERRETTI, A.; CALMON, M.; HIGA, R. Estoque e incremento de carbono em florestas e povoamentos de espécies arbóreas com ênfase na Floresta Atlântica do Sul do Brasil. Colombo: EMBRAPA Florestas, 2006. 165 p.

BRUN, E. J. Biomassa e nutrientes na Floresta Estacional Decidual, em Santa Tereza, RS. 2004. I 36 f. Dissertação (Mestrado em Engenharia Florestal) - Universidade Federal de Santa Maria, Santa Maria, 2004.

BRUN, F. G. K. Dinâmica do carbono orgânico em espécies arbóreas de uma floresta Estacional Decidual no Rio Grande do Sul. 2007. 106 f. Dissertação (Mestrado em Engenharia Florestal) - Universidade Federal de Santa Maria, Santa Maria, 2007.

CONSERVATION INTERNATIONAL DO BRASIL. Avaliação e ações prioritárias para a conservação da biodiversidade da Mata Atlântica e Campos Sulinos. Brasília: MMA/SBF, 2000. 40 p.

CORRÊA, G. F. Modelo de evolução e mineralogia da fração argila de solos do planalto de Viçosa. 1984. 87 f. Dissertação (Mestrado em Solos e Nutrição de Plantas) Universidade Federal de Viçosa, Viçosa, 1984.
DAVIS, L. S.; JOHNSON, K. N. Forest management. $3^{\text {rd }}$ ed. New York: McGraw-Hill Book, 1987. 790 p.

FERREIRA, R. L. C.; SOUZA, A. L.; REGAZZI, A. J. Dinâmica da estrutura de uma floresta secundária de transição: I., estrutura paramétrica. Revista Árvore, Viçosa, v. 22, n. I, p. 21-27, jan./fev. 1998.

FONSECA, W.; BENAYAS, J. R.; ALICE, F. E. Carbon accumulation in the biomass and soil of different aged secondary forests in the humid tropics of Costa Rica. Forest Ecology and Management, Amsterdam, v. 262, p. I400-I408, 20 II.

GANDOLFI, S.; LEITÃO-FILHO, H. F.; BEZERRA, C. L. Levantamento florístico e caráter sucessional de espécies arbustivo-arbóreas de uma floresta Mesófila Semidecídua no município de Guarulhos, SP. Revista Brasileira de Biologia, Rio de Janeiro, v. 55, n. 4, p. 753-767, 1995.

GRAYBILL, F. A. Theory and application of the linear model. Massachusetts: Duxbury, 1976. 704 p.

HOOVER, C. M.; LEAK, W. B.; KEEL, B. G. Benchmark carbon stocks from olg-growth forests in northern New England, USA. Forest ecology and Management, Amsterdam, v. 266, p. I08-II4, 2012.

MISSOURI BOTANICAL GARDEN. Tropicos MOBOT. Disponível em: <http://www.mobot.org/>. Acesso em: 10 dez. 20II.

RIBEIRO, S. C.; JACOVINE, L. A. G.; SOARES, C. P. B.; MARTINS, S. V.; SOUZA, A. L.; NARDELLI, A. M. B. Quantificação de biomassa e estimativa de estoque de carbono em uma floresta madura no município de Viçosa, Minas Gerais. Revista Árvore, Viçosa, v. 33, n. 5, p. 917-926, 2009.

SOUZA, A. L.; BOINA, A.; SOARES, C. P. B.; VITAL, B. R.; GASPAR, R. O.; LANA, J. M. Estoque e crescimento em volume, biomassa, carbono e dióxido de carbono em floresta Estacional Semidecidual. Revista Árvore, Viçosa, v. 35, n. 6, p. I277-I285, 201 I.

VELOSO, H. P.; RANGEL FILHO, A. L. R.; LIMA, J. C. A. Classificação da vegetação brasileira, adaptada a um sistema universal. Rio de Janeiro: IBGE, |99I. 123 p.

VIANELLO, R. L.; ALVES, A. R. Meteorologia básica e aplicações. Viçosa, MG: UFV, 1991. 448 p.

VIEIRA, S.; TRUMBORE, S.; CAMARGO, P. B.; SELHORST, D.; CHAMBERS, J. Q.; HIGUCHI, N.; MARTINELLI, L. A. Slow growth rates of Amazonian trees: consequences for carbon cycling. Proceedings of the National Academy of Sciences of the United States of America, Washington, v. I02, n. 5I, p. I8502-I8507, 2005.

WATZLAWICK, L. F; KIRCHNER, F. F; SANQUETTA, C. R.; SCHUMACHER, M. V. Fixação de carbon em Floresta Ombrófila Mista em diferentes estágios de regeneração. In: SANQUETTA, C. R.; WATZLAWICK, L. F.; BALBINOT, R.; ZILIOTTO, M. A. B.; GOMES, F. S. (Ed.). As florestas e o carbono. Curitiba: UFPR, 2002. p. I53-173. 